\title{
Comparison of suction above cuff and standard endotracheal tubes in neurological patients for the incidence of ventilator-associated pneumonia and in-hospital outcome: A randomized controlled pilot study
}

\author{
Sritam Jena, Sriganesh Kamath, Dheeraj Masapu, H. B. Veenakumari' ${ }^{1}$ Venkatapura J. Ramesh, \\ Varadarajan Bhadrinarayan, R. Ravikumar ${ }^{1}$
}

Background: Ventilator-associated pneumonia (VAP) is a common complication with endotracheal intubation. The occurrence of VAP results in significant mortality and morbidity. Earlier studies have shown reduction in the incidence of VAP with subglottic secretion drainage. The incidence ofVAP in neurologically injured patients is higher and can impact the neurological outcome.This study aimed to compare the incidence ofVAP with standard endotracheal tube (SETT) and suction above cuff endotracheal tube (SACETT) in neurologically ill patients and its impact on clinical outcome. Methods: Fifty-four patients with neurological illnesses aged $\geq 18$ years and requiring intubation and/or ventilation and anticipated to remain on ETT for $\geq 48 \mathrm{~h}$ were randomized to receive either SETT or SACETT. All the VAP preventive measures were similar between two groups except for the difference in type of tube. Results: The data of 50 patients were analyzed. The incidence of clinical VAP was $20 \%$ in SETT group and I2\% in SACETT group; $(P=0.70)$. The incidence of microbiologicalVAP was higher in the SETT group $(52 \%)$ as compared to SACETT group (44\%) but not statistically significant; $(P=0.78)$. There was no difference between the two groups for measured outcomes such as duration of intubation, mechanical ventilation, and Intensive Care Unit stay. Conclusions: In this pilot study in neurological population, a there was no significant difference in incidence of clinical and microbiological VAP was seen between SETT and SACETT, when other strategies for VAP prevention were similar. Other outcomes were similar with use of either tube for intubation.

Keywords: Mini-broncho-alveolar lavage fluid, neurological patients, outcome, subglottic secretions, ventilator-associated pneumonia

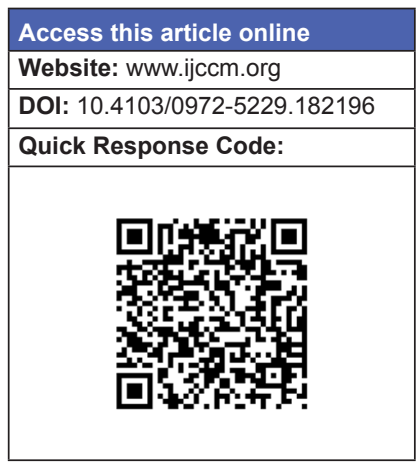

\section{Introduction}

Ventilator-associated pneumonia (VAP) is one of the important causes for morbidity and mortality in patients admitted to the Intensive Care Unit (ICU).

From:

Departments of Neuroanaesthesia and ${ }^{1}$ Neuromicrobiology, National Institute

of Mental Health and Neurosciences, Bengaluru, Karnataka, India

Correspondence:

Dr. Sriganesh Kamath, Department of Neuroanaesthesia, National Institute of Mental Health and Neurosciences, Bengaluru - 560 029, Karnataka, India.

E-mail: drsri23@rediffmail.com
The incidence of VAP in neurological patients is higher when compared to other medical and surgical

This is an open access article distributed under the terms of the Creative Commons Attribution-NonCommercial-ShareAlike 3.0 License, which allows others to remix, tweak, and build upon the work non-commercially, as long as the author is credited and the new creations are licensed under the identical terms.

\section{For reprints contact: reprints@medknow.com}

How to cite this article: Jena S, Kamath S, Masapu D, Veenakumari HB, Ramesh VJ, Bhadrinarayan $\mathrm{V}$, et al. Comparison of suction above cuff and standard endotracheal tubes in neurological patients for the incidence of ventilator-associated pneumonia and in-hospital outcome: A randomized controlled pilot study. Indian J Crit Care Med 2016;20:261-6. 
patient populations and has a significant impact on the neurological outcome. ${ }^{[1-3]}$ Peritubal leak and aspiration of the oropharyngeal secretions is primarily responsible for the occurrence of VAP. ${ }^{[4,5]}$ Therefore, removal of these secretions might lead to reduction in the incidence of VAP and thereby improvement in the outcome. The aim of this study was to compare the incidence of VAP and clinical outcome in neurologically ill patients with standard endotracheal tube (SETT) and suction above cuff endotracheal tube (SACETT) and to determine whether the incidence of VAP is reduced with the use of SACETT.

\section{Methods}

\section{Study design}

Sixty patients were eligible for recruitment into the study during the 24-month period, and six patients were excluded for nonfulfillment of the inclusion criteria. Following this, 54 patients were prospectively randomized to either the SETT or the specialized SACETT. The flow chart depicting the recruitment of patients into the study as per the CONSORT guidelines is shown in Figure 1. This study was conducted in the Neurological ICU (NICU) of tertiary neurosciences teaching hospital in Southern India during the period starting April 2013 till March 2015. Institutional Ethics Committee approval and written informed consent from the next of the kin of the patient were taken. During the study period, patients with neurological illnesses aged more than 18 years and requiring endotracheal intubation and/or mechanical ventilation for management in the NICU and anticipated to remain intubated for more than $48 \mathrm{~h}$ were screened for possible recruitment in this study. Patients who arrived to ICU already intubated or reintubated during the study period, patients with a tracheostomy and/or ventilator, and patients intubated for cardiac arrest or ventilated for $<48 \mathrm{~h}$ or in whom active care was likely to be withheld/withdrawn were excluded. The recruited patients were then randomized to receive either SETT or SACETT (Portex Blue Line SACETT; Smiths Medical ASD, Inc., Weston, MA, USA) based on a computer-generated randomization table.

\section{Neurological Intensive Care Unit management}

All randomized patients were screened daily for the occurrence of clinical VAP. The study protocol was maintained for at least 7 days of mechanical ventilation or tracheal extubation/tracheostomy, whichever was earlier. All the baseline investigations in the recruited patients were obtained within $24 \mathrm{~h}$ of intubation. Clinical diagnosis of VAP was based on the occurrence of pulmonary infection at least $48 \mathrm{~h}$ after intubation as evidenced by the presence of a new/progressive

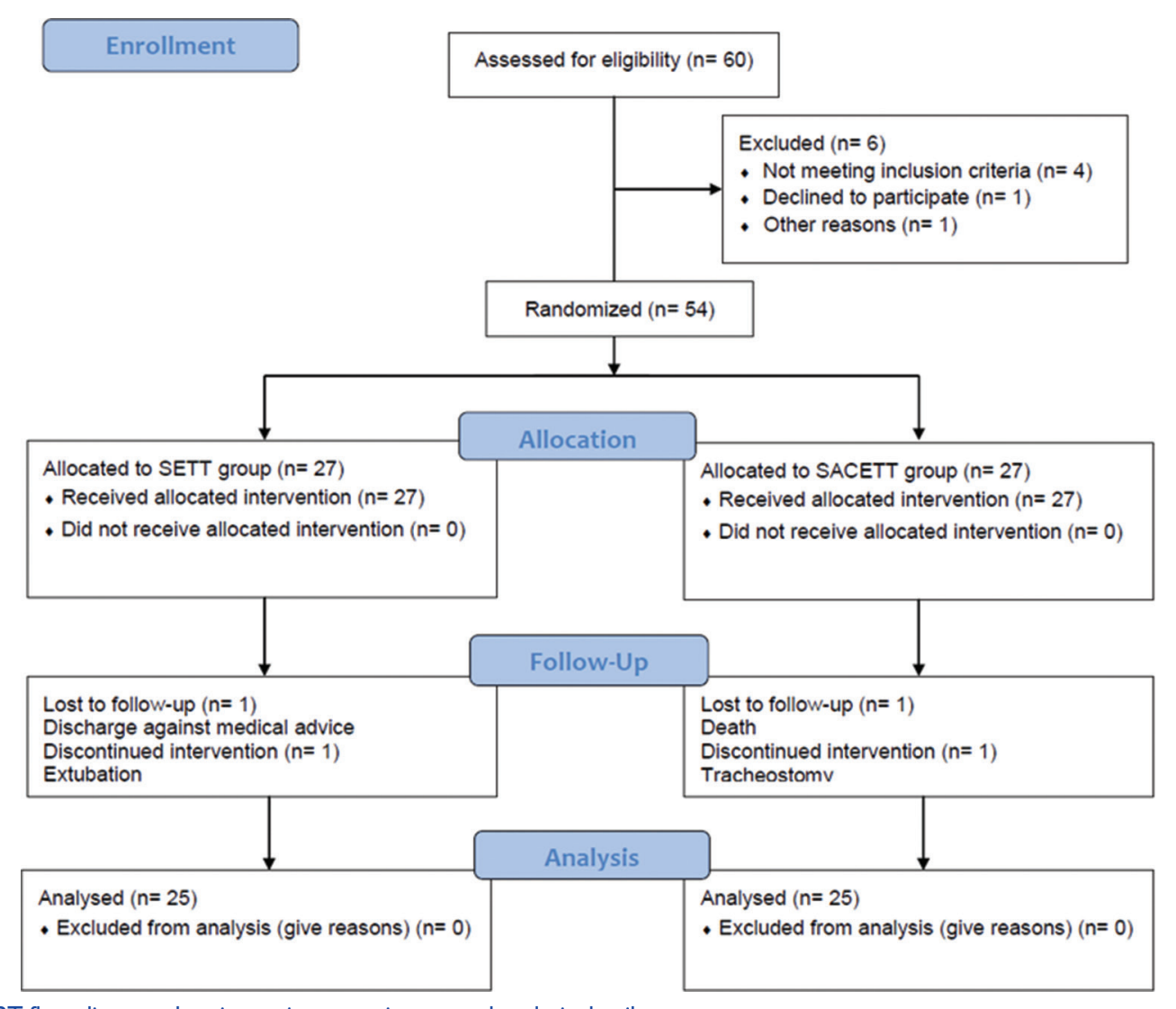

Figure I: CONSORT flow diagram showing patient recruitment and analysis details 
infiltrate on chest $\mathrm{X}$-ray, and at least one of the following criteria: Temperature $>38^{\circ} \mathrm{C}$ or $<36^{\circ} \mathrm{C}$, total leukocyte count $>11,000$ or $<4000$ cells $/ \mathrm{mm}^{3}$ plus new onset of purulent endotracheal secretions or change in character or volume of secretions, or worsening oxygenation as indicated by a decreased $\left(\mathrm{PaO}_{2} / \mathrm{FiO}_{2}\right)$ ratio. In addition, microbiological confirmation of VAP was done by analyzing the nonbronchoscopically performed bronchoalveolar lavage fluid (mini-BAL) as described by Rouby et al.${ }^{[6]}$ Mini-BAL samples were collected within $24 \mathrm{~h}$ of intubation and repeated not later than every $48 \mathrm{~h}$. All patients had at least three samples taken for analysis within $120 \mathrm{~h}$ of intubation. Samples were also obtained before any change or initiation of antibiotic therapy. The empirical antibiotic therapy was guided by our existing hospital policy. All the patients received similar modes of ventilation (controlled or assisted), and no neuromuscular blocking drugs were used to facilitate ventilation.

In the SACETT group, apart from the routine endotracheal suctioning to clear tracheobronchial secretions, the subglottic secretions were additionally suctioned manually with a $10 \mathrm{ml}$ syringe at an intended frequency of once every $2 \mathrm{~h}$ from the time of intubation. This specialized tube is designed with a port above the cuff for aspirating the oropharyngeal secretions collected above the cuff, thereby preventing potential peritubal leakage, and reduces the incidence of VAP. The number of subglottic secretion drainage (SSD) attempts and the amount of subglottic secretions collected in each patient in the SACETT group was prospectively recorded. SSD was not performed in the SETT group. Due to the nature of the intervention, physicians, and nurses were not blinded to the randomization; but the microbiologist was blinded to the study groups. Subglottic secretions were also sent for microbial analysis at the same time and were compared with mini-BAL microbial culture report. The incidence of clinical and microbiological VAP diagnosed and reported in this paper is based on the analysis of the data obtained in the first $120 \mathrm{~h}$ of intubation (early VAP).

\section{Other ventilator-associated pneumonia prevention strategies}

Except for the presence or absence of subglottic drainage of secretions, the other components of VAP prevention bundle including oral intubation, use of nasogastric tubes, early enteral nutrition, stress ulcer prophylaxis, maintenance of cuff pressure between 20 and $30 \mathrm{~cm} \mathrm{H}_{2} \mathrm{O}$ and semi-recumbent patient position were similarly adhered to during the study period in both groups. The other variables potentially influencing the occurrence of VAP were also recorded: Diagnosis, concomitant illness, preemptive antibiotic therapy, steroid drugs, blood transfusion, ventilatory modes, and type of humidification.

The primary endpoint of the study was the incidence of early-onset clinical and microbiological VAP in the SACETT and SETT groups. Secondary outcome variables were durations of intubation, mechanical ventilation, and ICU stay.

\section{Statistical analysis}

The quantitative variables are expressed as mean \pm standard deviation. Categorical variables are expressed as incidence or number and percentage. The SACETT and SETT groups were compared by analysis of variance test for continuous variables and by Chi-square or Fisher's exact test for categorical variables. $P<0.05$ was considered significant. The statistical analysis was performed using Statistical Package for the Social Sciences version 16 (SPSS Inc., Chicago, IL, USA).

\section{Results}

Of the 54 patients randomized, four patients were excluded from the analysis due to violation of protocol after randomization for reasons described in Figure 1. The results of the analysis performed in 50 patients are described. There was no difference between the groups for any demographic parameters [Table 1]. The neurological diagnoses of the study population predominately consisted of medically managed neurological patients and the distribution is shown in Figure 2. The incidence of clinical VAP in this study was 20\% $(5 / 25)$ in SETT group and $12 \%(3 / 25)$ in SACETT group; $(P=0.70)$ [Figure 3]. The incidence of microbiological VAP was higher in the SETT group $(13 / 25 ; 52 \%)$ as compared to the SACETT group $(11 / 25 ; 44 \%)$ but not statistically significant; $(P=0.78)$ [Figure 4]. The absolute reduction in the clinical and microbiological VAP was $8 \%$ each with use of SACETT. The relative risk reduction for clinical

Table I: Demographics and potential risk factors for ventilator-associated pneumonia

\begin{tabular}{|c|c|c|c|}
\hline \multirow[t]{2}{*}{ Parameters } & \multicolumn{2}{|c|}{ Mean \pm SD or $n$} & \multirow{2}{*}{$\begin{array}{c}\text { Significance } \\
(P)\end{array}$} \\
\hline & SETT $(n=25)$ & SACETT $(n=25)$ & \\
\hline Age (years) & $36.9 \pm 12.8$ & $42.2 \pm 17.9$ & 0.23 \\
\hline \multicolumn{4}{|l|}{ Gender } \\
\hline Male & 16 & 17 & 1.00 \\
\hline Co-morbidity & 4 & 4 & 0.65 \\
\hline Steroids & 5 & I & 0.19 \\
\hline Blood transfusion & I & 2 & 1.00 \\
\hline $\begin{array}{l}\text { Preemptive } \\
\text { antibiotics received }\end{array}$ & 17 & 18 & 0.5 \\
\hline
\end{tabular}

SETT: Standard endotracheal tube; SACETT: Suction above cuff endotracheal tube; SD: Standard deviation 


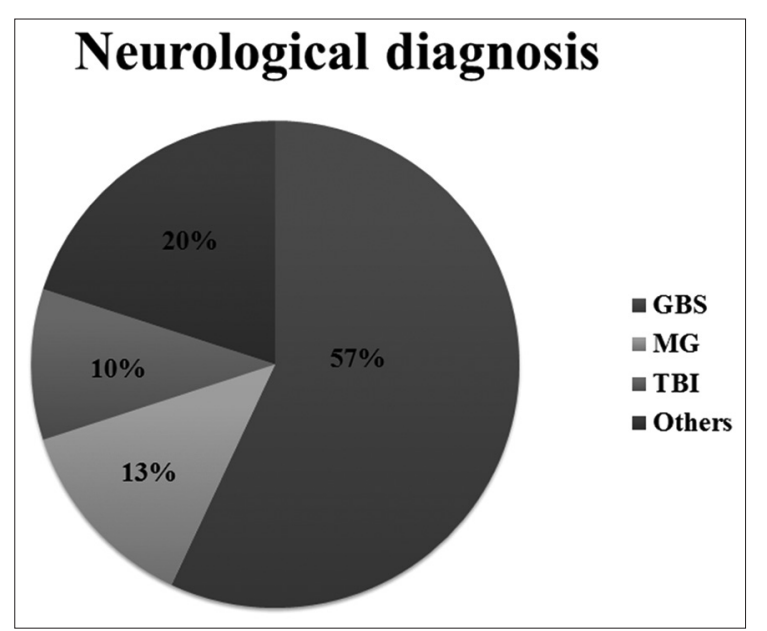

Figure 2: Neurological diagnoses of the study population and their distribution; GBS: Guillian-Barre syndrome; MG: Myasthenia gravis; TBI: Traumatic brain injury

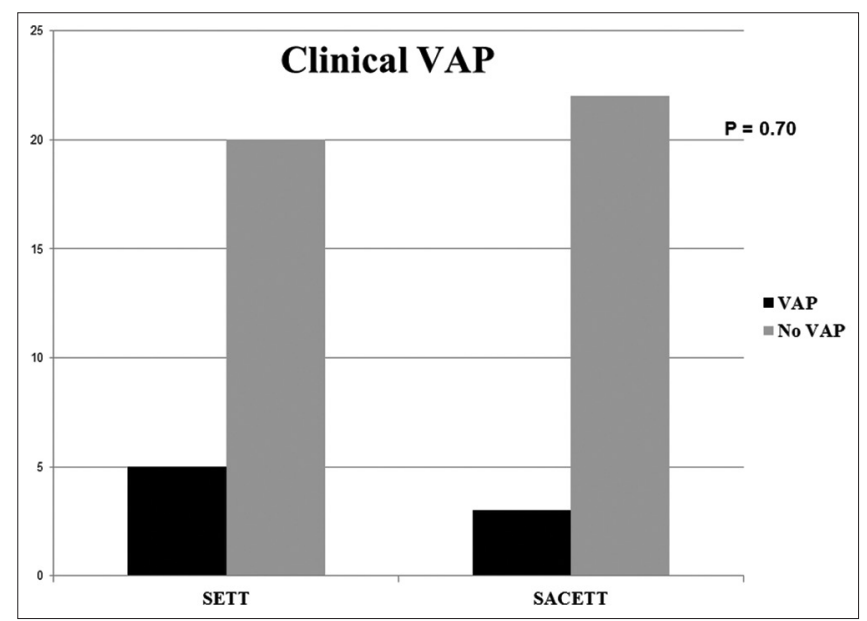

Figure 3: The incidence of clinical ventilator-associated pneumonia in patients with normal tube (standard endotracheal tube) and special tube (suction above cuff endotracheal tube) in this study

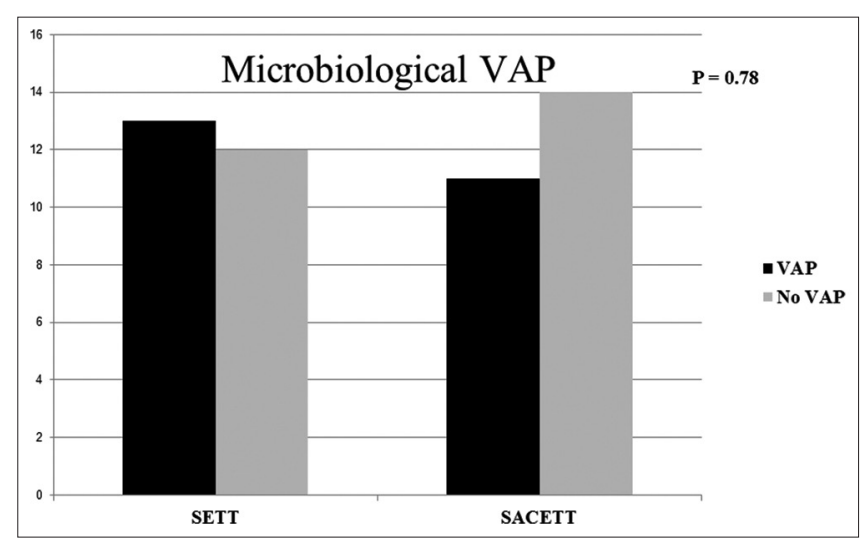

Figure 4: The incidence of microbiological ventilator-associated pneumonia in patients with normal tube (standard endotracheal tube) and special tube (suction above cuff endotracheal tube) in this study

VAP was $40 \%$ and for microbiological VAP was $15 \%$ with the use of SACETT. There was no difference in the potential risk factors studied (diagnosis, concomitant illness, preemptive antibiotic therapy, steroids, and blood transfusion) between the two groups. The ventilatory mode and type of humidification were similar in all the patients during the duration of the study. There was no difference between the two groups for measured outcomes such as duration of intubation, mechanical ventilation, and ICU stay [Table 2].

\section{Discussion}

The nosocomial infection rate in the NICU is very high. ${ }^{[7]}$ VAP is one of the most common nosocomial infections in the NICU and is associated with endotracheal intubation and mechanical ventilation. The reported incidence is between $8 \%$ and $28 \%$ in nonneurological population. ${ }^{[2]}$ Occurrence of VAP has been associated with significant mortality and morbidity, necessitating substantial utilization of resources in the patients managed in the intensive care setting despite preventive strategies being adopted. ${ }^{[2,3]}$ Aspiration of the oropharyngeal secretions and peritubal leak of the subglottic secretions are primarily responsible for the occurrence of VAP. ${ }^{[4,5]}$ Therefore, removal of these secretions must lead to reduction in the VAP rate and thereby improvement in the outcome. SSD using a specially designed ETT (SACETT) with a separate lumen above the cuff has shown to reduce the occurrence of VAP in some studies. ${ }^{[8,9]}$ However, a study in cardiac surgical patients examining the effect of continuous drainage of subglottic secretions has not shown difference in VAP rate. ${ }^{[10]}$ One reason for the differences in the incidence of VAP could be the heterogeneous study populations involving medical and surgical patients of different pathologies (sepsis, trauma, neurological, cardiac, pulmonary, etc.) in these studies. The current study, on the contrary, evaluated the incidence of VAP and its effect on outcome in only neurological patients, a homogeneous population. The incidence of VAP in neurologically injured patients is higher as compared to other population and has significant impact on the neurological outcome. Earlier studies involving neurological patients have documented an incidence of $28 \%$ in patients with stroke, ${ }^{[1]} 24 \%$ in patients with a mixed neurological diagnoses (stroke, trauma, and tumors), ${ }^{[11]} 45 \%$ in patients with traumatic brain injury ${ }^{[12]}$ and $78 \%$ in patients with GBS. ${ }^{[13]}$ The incidence of clinical VAP in our population was lower than that reported earlier, but the incidence of microbiological VAP was similar to the earlier studies involving neurological patients. The lower VAP rate in our study could be attributed to the strict adherence to all the components of VAP prevention bundle with the exception of study intervention (subglottic secretion drainage). In this 
Table 2: Outcome parameters in neurological patients

\begin{tabular}{lccc}
\hline Parameters (days) & \multicolumn{2}{c}{ Mean \pm SD } & Significance \\
\cline { 2 - 3 } & SETT $(\boldsymbol{n}=\mathbf{2 5})$ & SACETT $(\boldsymbol{n}=\mathbf{2 5})$ & $(\boldsymbol{P})$ \\
\hline Duration of intubation & $21.9 \pm 14.1$ & $24.9 \pm 19.6$ & 0.54 \\
Duration of ventilation & $17.2 \pm 11.5$ & $21.5 \pm 17.6$ & 0.31 \\
Duration of ICU stay & $24.4 \pm 14.2$ & $29.8 \pm 20.1$ & 0.28 \\
\hline
\end{tabular}

SETT: Standard endotracheal tube; SACETT: Suction above cuff endotracheal tube; SD: Standard deviation; ICU: Intensive Care Unit

study, the incidence of VAP, though reduced, was not statistically different with the use of SACETT questioning the utility and cost-effectiveness of this special tracheal tube and its impact on the outcomes in this population when employed on a routine basis.

The incidence of VAP also varies depending on how the sample is collected. Protected bronchial sampling and BAL are bronchoscopic guided techniques of obtaining samples and are considered to be more specific for VAP. However, these techniques are labor intensive, require the availability of a bronchoscope and an experienced operator and are not always possible in routine clinical setups in most ICUs. Moreover, the sensitivity of nonbronchoscopic techniques like mini-BAL is higher compared to bronchoscopic guided sample acquisition. ${ }^{[14]}$ Rouby et al. described the technique of blind mini-BAL for the first time, and it has been used widely since then, to diagnose VAP. ${ }^{[6]}$ This technique is user-friendly and yet provides a representative sample of bronchoalveolar secretions for the diagnosis of VAP. Hence, this is considered superior to the routinely obtained sample by suctioning of the endotracheal tube in ICU, which largely consists of tracheal secretions. Erden et al. have demonstrated that a small volume of $10-20 \mathrm{ml}$ of instilled fluid is sufficient to obtain good bronchial sample using blind mini-BAL technique for the diagnosis of nosocomial pneumonia and hence this technique was used in our study. ${ }^{[15]}$

The use of special tube for draining subglottic secretions did not lead to a different VAP incidence or outcome in the current study. The incidence of clinical and microbiological VAP though lower in patients having SACETT, was not statistically significant. The reason for this lack of difference in VAP and thereby outcome in patients with two different tracheal tubes could be the following. First, the cumulative volume of secretions aspirated from the subglottic region using the special tube was negligible (mean volume of $4.4 \mathrm{ml} / \mathrm{d}$ [range $0-14 \mathrm{ml} / \mathrm{d}]$ ). This probably resulted from the inability to aspirate thick and inspissated secretions through the small channel of the tube or from occlusion of the aspiration port of theSACETT by the mucus membrane of the trachea from the negative pressure during aspiration. Second, the volume of oropharyngeal secretions in both groups was not clinically different. Hence, in the absence of significant aspiration of secretions from the subglottic region, the peritubal leak of secretions in both groups would have been similar. Further, with strict adherence to rest of the VAP prevention protocols, the additional benefit from placement of a special tube was not visible in this sample of patients. Finally, in this study, of the 11 patients who developed microbiological VAP in the SACETT group, only two patients had the same organism coinciding with the subglottic organisms. This suggests that the subglottic organisms may not have contributed to the VAP in majority of the cases in this population.

Many earlier studies have shown SSD to reduce the incidence of VAP. Lacherade et al. in a mixed medical and surgical ICU population observed that intermittent SSD using the special tube as compared to conventional ETT resulted in a significant reduction of early microbiological VAP ( $<5$ days). However, this reduction in VAP with SSD did not translate into benefit with respect to outcomes such as duration of mechanical ventilation or hospital mortality. ${ }^{[16]}$ On the contrary, a large systematic review involving 13 randomized controlled trials consisting of 2442 patients concluded that SSD is effective in preventing VAP in patients at risk and also reduced the ventilation duration and ICU stay ${ }^{[17]}$ Both the continuous and intermittent drainage of the subglottic secretions have been shown to be equally effective in reduction of VAP. ${ }^{[17]}$ However, in an in vitro study comparing Hi-Lo Evac, Teleflex ISIS, and Portex Blue Line SACETT, the ability to aspirate subglottic secretions either continuously or intermittently was least effective with Portex Blue Line SACETT due to mucus obstruction of the aspirating port. ${ }^{[18]}$ In the current study, in neurological population using SACETT, similar difficulty in aspirating subglottic secretions was seen. This reflected in the failure to demonstrate superiority of SACETT in reducing VAP or improving outcome in mechanically ventilated patients compared to SETT.

The strength of this study is that the studied population was homogeneous with respect to diagnosis and pathology (all neurological patients). We evaluated both clinical VAP and microbiological VAP with sample analyzed using mini-BAL. Furthermore, all aspects of VAP prevention were similar between the two groups except for the SSD. The major limitation of this study is the small sample size. Based on this pilot study, the number need to treat (NNT), or the number of patients that need to be treated with SACETT for one benefit (reduction in VAP) to occur compared with SETT was found to be 12 . 
NNT is an epidemiological measure for assessing the effectiveness of a health-care intervention, in this case, a costly specialized tube. Since this number is high, the effectiveness of this intervention (SACETT) appears to be very low. The second limitation is that we did not calculate the effect of late-onset VAP. This analysis would have been flawed as majority of the patients included in the study underwent tracheostomy early, and all received a similar tracheostomy tube without the facility for drainage of subglottic secretions. In view of the findings of this study, the cost (SACETT costs about 5.77 times the SETT) and difficulty involved in predicting which patient is likely to develop VAP to recommend the selective use of this special tube, the overall benefit remains questionable.

\section{Conclusion}

In this small homogeneous neurological population, there was a trend towards in both clinical and microbiological VAP with the use of SACETT for intubation compared to SETT when other strategies for VAP prevention were similar between the groups, but this was not statistically significant. Other outcome parameters were not different between the two groups. Our data provide evidence for a future prospective study where the sample size needed to test for a difference can be determined from the results of this study. In addition, future studies should address the clinical and cost effectiveness of SSD with a better tube design in a larger neurological cohort.

\section{Financial support and sponsorship}

Departmental, SACETT tubes for the study were provided by Smiths Medical India Pvt Ltd.

\section{Conflicts of interest}

There are no conflicts of interest.

\section{References}

1. Kasuya Y, Hargett JL, Lenhardt R, Heine MF, Doufas AG, Remmel KS, et al. Ventilator-associated pneumonia in critically ill stroke patients: Frequency, risk factors, and outcomes. J Crit Care 2011;26:273-9.
2. Chastre J, Fagon JY. Ventilator-associated pneumonia. Am J Respir Crit Care Med 2002;165:867-903.

3. Klompas M. Does this patient have ventilator-associated pneumonia? JAMA 2007;297:1583-93.

4. American Thoracic Society; Infectious Diseases Society of America. Guidelines for the management of adults with hospital-acquired, ventilator-associated, and healthcare-associated pneumonia. Am J Respir Crit Care Med 2005;171:388-416.

5. Oikkonen M, Aromaa U. Leakage of fluid around low-pressure tracheal tube cuffs. Anaesthesia 1997;52:567-9.

6. Rouby JJ, Rossignon MD, Nicolas MH, Martin de Lassale E, Cristin S, Grosset J, et al. A prospective study of protected bronchoalveolar lavage in the diagnosis of nosocomial pneumonia. Anesthesiology 1989;71:679-85.

7. Li HY, Li SJ, Yang N, Hu WL. Evaluation of nosocomial infection risk using APACHE II scores in the neurological intensive care unit. J Clin Neurosei 2014;21:1409-12.

8. Mahul P, Auboyer C, Jospe R, Ros A, Guerin C, el Khouri Z, et al. Prevention of nosocomial pneumonia in intubated patients: Respective role of mechanical subglottic secretions drainage and stress ulcer prophylaxis. Intensive Care Med 1992;18:20-5.

9. Smulders K, van der Hoeven H, Weers-Pothoff I, Vandenbroucke-Grauls C. A randomized clinical trial of intermittent subglottic secretion drainage in patients receiving mechanical ventilation. Chest 2002;121:858-62.

10. Kollef MH, Skubas NJ, Sundt TM. A randomized clinical trial of continuous aspiration of subglottic secretions in cardiac surgery patients. Am J Respir Crit Care Med 1999;116:1339-46.

11. Prendergast V, Hallberg IR, Jahnke H, Kleiman C, Hagell P. Oral health, ventilator-associated pneumonia, and intracranial pressure in intubated patients in a neuroscience intensive care unit. Am J Crit Care 2009; 18:368-76.

12. Zygun DA, Zuege DJ, Boiteau PJ, Laupland KB, Henderson EA, Kortbeek JB, et al. Ventilator-associated pneumonia in severe traumatic brain injury. Neurocrit Care 2006;5:108-14.

13. Orlikowski D, Sharshar T, Porcher R, Annane D, Raphael JC, Clair B. Prognosis and risk factors of early onset pneumonia in ventilated patients with Guillain-Barré syndrome. Intensive Care Med 2006;32:1962-9.

14. Papazian L, Thomas P, Garbe L, Guignon I, Thirion X, Charrel J, et al. Bronchoseopic or blind sampling techniques for the diagnosis of ventilator-associated pneumonia. Am J Respir Crit Care Med 1995;152 (6 Pt 1):1982-91.

15. Erden V, Basaranoglu G, Beycan I, Delatioglu H, Hamzaoglu NS. Reproducibility of mini-BAL culture results using $10 \mathrm{ml}$ or $20 \mathrm{ml}$ instilled fluid. Intensive Care Med 2003;29:1856.

16. Lacherade JC, De Jonghe B, Guezennec P, Debbat K, Hayon J, Monsel $\mathrm{A}$, et al. Intermittent subglottic secretion drainage and ventilator-associated pneumonia: A multicenter trial. Am J Respir Crit Care Med 2010;182:910-7.

17. Muscedere J, Rewa O, McKechnie K, Jiang X, Laporta D, Heyland DK Subglottic secretion drainage for the prevention of ventilator-associated pneumonia: A systematic review and meta-analysis. Crit Care Med 2011;39:1985-91.

18. Mujica-Lopez KI, Pearce MA, Narron KA, Perez J, Rubin BK. In vitro evaluation of endotracheal tubes with intrinsic suction. Chest 2010;138:863-9. 